\title{
Évaluation du rôle des facteurs physicochimiques dans la clarification du lactosérum
}

\author{
A Pierre 1, Y Legraet 1, J Fauquant 1, \\ M Piot 1, C Durier 2, A Kobilinsky 2 \\ 1 INRA, laboratoire de recherches de technologie laitière, 65, rue de St-Brieuc, 35042 Rennes; \\ 2 INRA, CNRA, laboratoire de biométrie, route de St-Cyr, 78026 Versailles, France
}

(Reçu le 24 février 1992; accepté le 17 avril 1992)

\begin{abstract}
Résumé - L'influence des différents facteurs intervenant lors de la clarification d'un lactosérum d'emmental a été étudiée à l'aide d'un plan d'expérience. Les facteurs étaient : la teneur en protéines totales $\left(8-50 \mathrm{~g}^{\circ} \mathrm{kg}^{-1}\right)$, la teneur en calcium $\left(0,4-4,2 \mathrm{~g}^{\circ} \mathrm{kg}^{-1}\right)$, la température initiale (2-

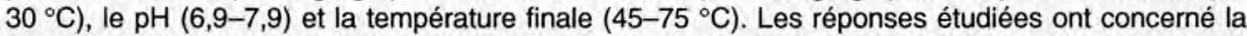
turbidité (DO), les teneurs résiduelles en $\mathrm{Ca}, \mathrm{P}, \mathrm{Mg}, \mathrm{K}$, la matière azotée totale (MAT), et le $\mathrm{pH}$, en fonction des facteurs décrits. ci-dessus pris individuellement ou en interaction par paires. Les effets linéaires et quadratiques calculés indiquent que les facteurs intervenant sur la DO sont, par ordre d'importance décroissante, la teneur en calcium et la température finale; la température initiale et le $\mathrm{pH}$ n'ont pas d'influence significative; la teneur en protéines totales a un effet dépressif sur la clarification. La clarification a été possible pour les lactosérums à toutes les teneurs en protéines étudiées; une représentation graphique des conditions de clarification est donnée pour 5 concentrations. La taille des particules du lactosérum responsables de l'opalescence a été estimée par diffusion quasi élastique de la lumière. Des particules de $105-512 \mathrm{~nm}$ de diamètre ont été déterminées dans le lactosérum brut. Dans le lactosérum clarifié, seules des petites particules de $8 \mathrm{~nm}$ ont été détectées. La nature et la composition de ces différentes particules n'a pu être élucidée. La clarification entraîne la précipitation du $\mathrm{Ca}$ et du $\mathrm{P}$ minéral et organique; la précipitation des peptides phosphorylés de la caséine $\beta$ constituant les protéoses peptones du lactosérum a été démontrée. La mise en évidence de la précipitation des membranes de globules gras n'a pas été réalisée, mais de nombreux phospholipides solubles sont encore présents dans le lactosérum après clarification.
\end{abstract}

\section{lactosérum / clarification}

Summary - Optimization of physico-chemical factors for whey clarification. Computer analysis of a surface experimental design evaluated the role of 5 factors, eg total protein content (8$50 \mathrm{~g}^{\circ \mathrm{kg}^{-1}}$ ), calcium level $\left(0.4-4.2 \mathrm{~g}^{\circ} \mathrm{kg}^{-1}\right)$, initial temperature $\left(2-30{ }^{\circ} \mathrm{C}\right), \mathrm{pH}(6.9-7.9)$ and final temperature $\left(45-75^{\circ} \mathrm{C}\right)$ in clarification of Emmental whey. Response surface plots were generated for turbidity (OD; optical density), $\mathrm{Ca}, \mathrm{P}, \mathrm{Mg}, \mathrm{K}, \mathrm{MAT}, \mathrm{pH}$ as functions of protein content versus calcium level, or final temperature versus calcium level, or final temperature alone. Ranking of the estimated linear and quadratic effects indicated that the order of decreasing importance of the different factors 
upon optical density in Emmental clarified whey was initial calcium level followed by final temperature; initial temperature and $\mathrm{pH}$ had no significant effects; total protein content had a depressive effect on clarification. Clarification of whey was performed for all the protein contents investigated. A graphic representation of the optimal values of factors is presented for whey at 5 protein contents. Particle size of whey colloidal material responsible for light diffusion and opacity was measured by light scattering. Particles of 105-512 nm in diameter were predominant. In clarified whey, they disappeared and only small particles of $8 \mathrm{~nm}$ diameter were detected. The nature and composition of the different particles were not elucidated. Chemical analysis of treated whey and electrophoresis of proteins provided data for estimating the components in the precipitated material. Precipitation involved $\mathrm{Ca}, \mathrm{P}$ including mineral $P_{4}$ and organic $P$. The precipitation of $\beta$-casein phosphopeptides of whey (PP5 and PP8) was demonstrated. The demonstration of milk fat globule membrane precipitation was not carried out; it was, however, observed that phospholipids were still present in clarified whey (qualitative analysis).

\section{whey / clarification}

\section{INTRODUCTION}

Avec le développement de l'ultrafiltration du lactosérum s'est posé le problème de l'optimisation des flux de perméation. De nombreux procédés de prétraitement ont été proposés pour diminuer l'effet colmatant des lactosérums (Hayes et al, 1974; Marshall et al, 1974; Lee et Merson, 1976). Une agrégation partielle des constituants colmatants permet leur rétention par la membrane avec une amélioration des flux, mais ils sont alors présents dans la fraction protéique que l'on souhaite purifier. L'élimination complète du précipité préalablement à l'ultrafiltration a également été proposée en utilisant différents procédés de clarification du lactosérum : la décantation (de Wit et al, 1978; Fauquant et al, 1985a; Wasen et Lehmann, 1989), la centrifugation (Fauquant et al, 1985b) ou la microfiltration (Maubois et al, 1987). Le lactosérum clarifié obtenu est un liquide limpide et moins colmatant (Daufin et al, 1991, 1992a, b).

La composition de la fraction précipitée lors de la clarification varie évidemment selon le lactosérum mis en œuvre et selon le protocole utilisé. Mais, on y trouve toujours, en quantités relatives différentes, des lipides (8-28\%), dont un tiers de lipides polaires (Baumy et al, 1990; Ducruet et al, 1990), des matières azotées (8$28 \%$ ), constituées de caséines associées à de la $\beta$-lactoglobuline (Hayes et al, 1974), du calcium, $7-41 \%$ et du phosphore, 18$23 \%$ (Fauquant et al, 1985a, b).

Les mécanismes de la précipitation sont complexes et mal connus. Les minéraux précipitent à l'état de phosphate de calcium et de citrate de calcium (Hayes et al, 1974). Dans le procédé de de Wit et al (1978), les lipides sont déstabilisés dans des conditions de $\mathrm{pH}$ et de force ionique bien définies. Dans les autres procédés, les conditions de précipitation des fractions lipidiques dépendent des caractéristiques physicochimiques des lactosérum.

Afin de mieux comprendre l'influence des conditions opératoires, nous avons réalisé une étude systématique de la clarification d'un lactosérum d'Emmental en fonction de 5 facteurs mis en jeu lors de ces traitements : concentration en protéines par ultrafiltration, température du lactosérum, teneur en calcium initiale du lactosérum, $\mathrm{pH}$ et température lors du chambrage. Les expérimentations ont été conduites selon un plan permettant de réaliser une modélisation statistique de l'in- 
fluence respective de ces 5 facteurs. Nous avons suivi les modifications physicochimiques du lactosérum, induites par le traitement afin de déterminer les constituants précipités et d'en déduire les mécanismes physico-chimiques mis en jeu lors de la clarification.

\section{MATÉRIEL ET MÉTHODES}

\section{Lactosérum}

Le lactosérum provenait d'une fabrication d'Emmental (ULN, Montauban-de-Bretagne, France). II était filtré sur un tamis de $20 \mu \mathrm{m}$ et écrémé à $50{ }^{\circ} \mathrm{C}$ (Westphalia MSD 300; $9000 \mathrm{~g}$ ) à l'usine avant le prélèvement.

\section{UItrafiltration}

Sept cents litres de lactosérum étaient ultrafiltrés sur un module Pléiade Rhône-Poulenc, équipé de membranes IRIS 3038 (seuil de coupure $20 \mathrm{kDa}$; aire membranaire $4 \mathrm{~m}^{2}$ ) à $50^{\circ} \mathrm{C}$ et avec un flux moyen de $150 \quad 1 \cdot \mathrm{h}^{-1} \cdot \mathrm{m}^{-2}$. La concentration était conduite jusqu'à un facteur de concentration volumique (FCV) de 8. Le FCV était calculé à partir du volume de lactosérum mis en œuvre, $V L$, et du volume de rétentat obtenu, VR $(F C V=V L V R)$. En vue de préparer des échantillons de lactosérum à différents $F C V$, le rétentat final était dilué dans les proportions voulues avec de l'ultrafiltrat moyen. $\mathrm{Ce}$ mode opératoire a été choisi car il permettait d'obtenir des lactosérums à une teneur en MAT prédéterminée. Les échantillons étaient stockès jusqu'au lendemain $\left(15 \mathrm{~h}\right.$ à $\left.2^{\circ} \mathrm{C}\right)$, jour où l'expérimentation était réalisée.

\section{Traitements du lactosérum}

Les études ont été réalisées sur des fractions aliquotes de $100 \mathrm{ml}$ de lactosérum. La température initiale (Ti) du lactosérum était ajustée par immersion des échantillons au bain de glace ou au bain-marie, selon la température à obtenir.
La température finale de chambrage était obtenue en plaçant les échantillons dans un bainmarie régulé à une température supérieure de $2^{\circ} \mathrm{C}$ à la température à atteindre par l'échantillon. La durée de montée en température était de $2 \mathrm{~min}$. L'addition de calcium (Ca) était faite en utilisant une solution de $\mathrm{CaCl}_{2} 4 \mathrm{~mol} \cdot \mathrm{l}^{-1}$. Le $\mathrm{pH}$ était ajusté avec $\mathrm{NaOH} 5 \mathrm{~N}$.

\section{Protocole de clarification}

La clarification comportait les étapes suivantes : équilibrage à la température initiale (Ti), addition éventuelle de calcium (Ca), ajustement du $\mathrm{pH}$ $(p H i)$, chambrage à la température finale $(T f)$, pendant $10 \mathrm{~min}$, centrifugation (centrifugeuse Heraeus $2000 \mathrm{~g} ; 15 \mathrm{~min}, 30^{\circ} \mathrm{C}$ ) afin de sédimenter les constituants du lactosérum déstabilisés. Les surnageants étaient collectés, puis analysés.

\section{Analyses chimiques}

La turbidité des surnageants était estimée par la mesure de la densité optique (DO) à $600 \mathrm{~nm}$, après une dilution au $1 / 5$ dans de l'eau distillée, en utilisant un spectrophotomètre optique Kontron (Uvicon 810). La matière azotée totale (MAT) était dosée en utilisant un Dairy Lab IR, avec un étalonnage sur lactosérum. Le dosage du $\mathrm{Ca}$, du $\mathrm{K}$ et du $\mathrm{Mg}$ était fait par spectrophotométrie d'absorption atomique, le dosage du $\mathrm{P}$, selon la norme FIL $n^{\circ} 33$ (1982).

L'estimation des constituants du lactosérum retenus par la membrane d'ultrafiltration a été faite à partir de la teneur du constituant dans le lactosérum ( $\left.X_{\mathrm{LS}}\right)$ au $F C V 1$ et dans le rétentat $\left(X_{\mathrm{R}}\right)$ au $F C V_{\mathrm{R}}\left(F C V_{\mathrm{R}}=7\right)$ : la quantité retenue par kg de lactosérum est :

$$
Q r=\left(X_{\mathrm{R}}-X_{\mathrm{LS}}\right) / F C V_{\mathrm{R}}-1
$$

Cette valeur peut également être rapportée à la teneur initiale totale dans le lactosérum, pour évaluer le pourcentage du constituant retenu :

$$
\%=\operatorname{Qr} \times 100 / X_{\mathrm{LS}}
$$

La recherche qualitative des phospholipides présents dans les échantillons était réalisée par 
chromatographie sur couche mince. L'extraction des phospholipides était faite selon le protocole de Folch et al (1957), modifié par Bligh et Dyer (1959) à partir d'échantillons lyophilisés, et la séparation selon la méthode de Mangold et Kammereck (1962).

L'analyse qualitative des protéines était réalisée par électrophorèse, soit selon le protocole de Andrews (1983) soit en présence de SDS, selon Laemmli (1970).

La détermination de la taille des particules en suspension était effectuée par diffusion quasi élastique de la lumière sur un appareil N4MD de Coultronics. Les mesures ont été faites à $20^{\circ} \mathrm{C}$, sur des échantillons dilués dans de l'ultrafiltrat synthétique (Jenness et Koops, 1962) et sous un angle de $90^{\circ}$ par rapport au faisceau incident. Les distributions de taille en intensité étaient converties en distributions en poids à partir d'un programme prenant en compte des particules sphériques (valeur des constantes de conversion $\alpha=32.10^{-6} ; B=$ $0,33)$.

\section{Plan d'expérience}

Les expérimentations étaient conduites de manière à recouvrir un large domaine de variation des facteurs étudiés. Elles permettent l'ajustement de modèles polynômiaux reliant les variables mesurées aux facteurs et une présentation des résultats sous forme de graphiques (tracé des surfaces de réponse). Trente-deux traitements étaient réalisés pour chaque expérimentation. Les facteurs et les niveaux retenus sont reportés dans le tableau I. Les valeurs des facteurs ainsi que leurs domaines de variation avaient été déterminées au cours d'essais préliminaires. Les échantillons étaient traités en ordre aléatoire.

Le plan d'expérience est un plan factoriel composite centré à 5 facteurs (Box et Wilson, 1951; Box et Hunter, 1957). Ces facteurs sont: 1) la concentration du lactosérum en protéines représentée par le facteur de concentration volumétrique de l'ultrafiltration $(F C V), 2)$ la température initiale de l'échantillon (Ti), 3) la teneur en calcium total (Ca) qui est la somme du calcium naturel et du calcium ajouté, 4) le pHauquel est ajusté l'échantillon, après addition de calcium $(\mathrm{pHi}), 5$ ) la température finale à laquelle est alors porté l'échantillon (Tf).

Les réponses mesurées dans les surnageants recueillis après la déstabilisation et la centrifugation étaient : densité optique (DO), matière azotée totale (MAT), pH, calcium (Ca) et magnésium $(\mathrm{Mg})$ potassium $(K)$ et phosphore (P).

Les effets factoriels ont été estimés par régression en ajustant un modèle polynominal de degré 2 aux données expérimentales (Noël et al, 1991) grâce au logiciel APOLOR (Durier et Kobilinski, 1989).

À partir de ces modèles, des surfaces de réponse ont été tracées sous forme de courbes d'iso-contours.

Tableau I. Niveaux des facteurs retenus pour les traitements.

Factor levels used for whey treatment.

Facteurs

Niveaux codés

\begin{tabular}{lllll}
\hline-2 & -1 & 0 & +1 & +2 \\
\hline
\end{tabular}

$\begin{array}{lccccc}F C V & 1 & 2,5 & 4 & 5,5 & 7 \\ T i\left({ }^{\circ} \mathrm{C}\right) & 2 & 10 & 16 & 22 & 30 \\ \mathrm{Ca}\left(\mathrm{kg}^{-1}\right) & 0,4 & 1,3 & 2,3 & 3,3 & 4,2 \\ \mathrm{pH} & 6,9 & 7,2 & 7,4 & 7,6 & 7,9 \\ \mathrm{Tf}\left({ }^{\circ} \mathrm{C}\right) & 45 & 53 & 60 & 67 & 75\end{array}$




\section{RÉSULTATS}

\section{Domaine de clarification}

La composition des lactosérums mis en œuvre et des rétentats est donnée dans le tableau II. La teneur en MAT des échantillons variait de 8,7 à $50,8 \mathrm{~g}^{\circ} \mathrm{kg}^{-1}$. Les teneurs en minéraux sont plus élevées dans les rétentats après ultrafiltration. L'augmentation atteint $37 \%$ pour $\mathrm{Ca}$ et $16 \%$ pour $\mathrm{K}$. Les pourcentages de minéraux $\mathrm{du}$ lactosérum retenus par la membrane sont respectivement de 2,6, de 6 et de 4,6\% pour $\mathrm{K}, \mathrm{Ca}$ et $\mathrm{P}$. La teneur en $\mathrm{P}$ retenue par la membrane représente $20 \mathrm{mg}^{\circ} \mathrm{kg}^{-1}$ de lactosérum.

Les lactosérums ont été traités dans les conditions décrites pour la réalisation du plan et les surnageants recueillis ont été analysés. Le tableau III donne les modèles significatifs à $95 \%$. La $\mathrm{Ti}$ et le $\mathrm{pH}$. sont sans influence significative sauf sur le $\mathrm{pH}$ final.

À partir de ces données, des graphiques ont été tracés présentant les réponses obtenues en fonction de 2 fac-. teurs, les autres facteurs étant fixés. Par exemple, la figure 1a montre la variation de $D O$ en fonction de $F C V$ en ordonnée et de $\mathrm{Ca}$ en abcisse. Les autres facteurs sont constants, $\mathrm{Tf}=60^{\circ} \mathrm{C}, \mathrm{pHi}=7,4, \mathrm{Ti}=$ $16^{\circ} \mathrm{C}$. On remarque une plage de DO minimale pour une teneur en $\mathrm{Ca}$ comprise entre 0,4 et $3,3 \mathrm{~g} \cdot \mathrm{kg}^{-1}$. La valeur seuil de $\mathrm{Ca}$ augmente avec FCV.

Une augmentation de la température de chambrage $\mathrm{Tf}$ dans l'intervalle $45-60{ }^{\circ} \mathrm{C}$ favorise la clarification (fig 1b); au-delà de cette valeur, on ne note pas d'effet de ce facteur dans les conditions représentées, $F C V=4, C a=2,3 \mathrm{~g}^{\circ \mathrm{kg}^{-1}}$.

Au travers de l'influence du facteur Tf sur la $D O$ (fig 1b), il apparaît que la clarification est un phénomène progressif, puis-

Tableau II. Composition du lactosérum mis en œuvre et des rétentats d'ultrafiltration. Protein and mineral content of whey and ultrafiltrated wheys.

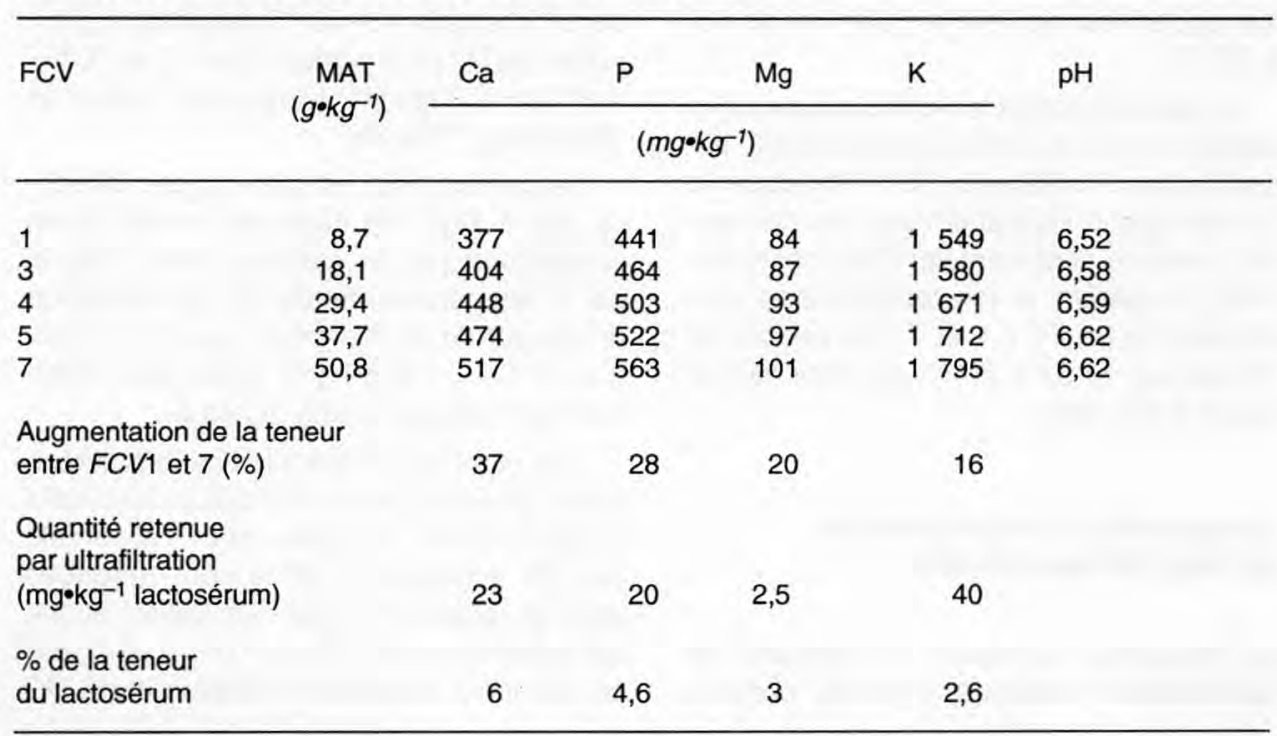


Tableau III. Modèles significatifs à $95 \%$. Ecart type résiduel $=E T R$. Model at $95 \%$ confidence level; standard deviation : ETR.

$$
\begin{aligned}
& D O=0,5+1,3 \mathrm{FCV}-1,7 \mathrm{Ca}-1.2 \mathrm{Tf}-1,2 \mathrm{FCV} . \mathrm{Ca}+1,1 \mathrm{Ca}^{2}+0,8 \mathrm{Tf}^{2} \quad E T R=1,6
\end{aligned}
$$

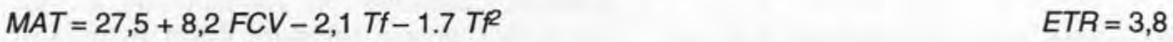

$$
\begin{aligned}
& \mathrm{Ca}=1798,2+85,1 \mathrm{FCV}=694,5 \mathrm{Ca}-96,9 \mathrm{Tf}-95,3 \mathrm{FCV} . \mathrm{Ca} \quad \text { ETR }=151,9 \\
& P=178,8+64,9 \mathrm{FCV}-82,2 \mathrm{Ca}-42,5 \mathrm{Tf}-53,2 \mathrm{FCV} . \mathrm{Ca}+43,9 \mathrm{Ca}^{2}+29,3 \text { Tf } \quad E T R=63,2 \\
& M g=86,1+4,6 \text { FCV }-0,5 \mathrm{Ca}-3,1 \mathrm{FCV} . \mathrm{Ca} \quad E T R=4,1 \\
& \begin{array}{ll}
K=1633+45,8 \mathrm{FCV}-11,7 \mathrm{Ca}+9.5 \mathrm{Tf} & E T R=20,7
\end{array} \\
& \mathrm{pH}=7,0+0,05 \mathrm{Ti}+0,1 \mathrm{pHi}-0,04 \mathrm{Ca} \quad E T R=0,08
\end{aligned}
$$

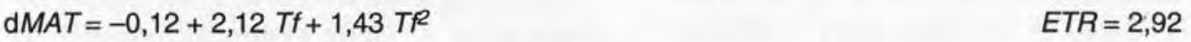

$$
\begin{aligned}
& \mathrm{d} P=341,6-54,1 F C V+81,8 \mathrm{Ca}+42,9 \mathrm{Tf}-20,3 F C V^{2}+52,6 \mathrm{i} \\
& -44,0 \mathrm{Ca}^{2}-29,4 T R^{2} \quad \text { ETR }=44,2 \\
& \mathrm{dCa}=604,9-120,7 \mathrm{FCV}+289,5 \mathrm{Ca}+96,9 \mathrm{Tf}+99,3 \mathrm{FCV} \cdot \mathrm{Ca}-61,5 \mathrm{Tf} \quad \quad E T R=118,8
\end{aligned}
$$

qu'il existe des conditions de traitement permettant d'obtenir des DO intermédiaires, donc des clarifications partielles. Dans les conditions optimales, la DO du surnageant est de 0,07 à $F C V 1$ et de 0,5 à FCV 7.

La figure 2 définit les combinaisons des facteurs $\mathrm{Tf}$ et $\mathrm{Ca}$ conduisant à des $\mathrm{DO}$ minimales pour différents FCV. On observe que lorsque le FCV augmente, les courbes de niveau se déplacent vers les concentrations en calcium et les températures plus élevées. Entre $F C V 1$ et 7 , les valeurs de Tf passent de 53 à $60^{\circ} \mathrm{C}$ et celles de $\mathrm{Ca}$ de 0,6 à $3,1 \mathrm{~g} \mathrm{~kg}^{-1}$.

\section{Composition physico-chimique des lactosérums clarifiés}

Le phosphore présente un domaine de concentration minimale (fig $3 a$ ) compris entre 100 et $150 \mathrm{mg}^{\circ} \mathrm{kg}^{-1}$ dans la plage de variation du $\mathrm{Ca}$ de 0,4 à $3,3 \mathrm{~g}^{\circ} \mathrm{kg}^{-1}$; cette teneur en $\mathrm{Ca}$ est d'autant plus haute que le FCV est élevé. La température finale $T f$, dans l'intervalle $45-60^{\circ} \mathrm{C}$ favorise la diminution du $\mathrm{P}\left(14,6 \mathrm{mg}^{\circ} \mathrm{kg}^{-1} \mathrm{par}^{\circ} \mathrm{C}\right.$, à $\mathrm{FCV}=$ 4 et $C a=2,3 \mathrm{~g}^{\circ} \mathrm{kg}^{-1}$ ) jusqu'à une valeur de $160 \mathrm{mg}^{\circ} \mathrm{kg}^{-1}$ (fig 3b).

Le calcium final est peu ou pas influencé par le FCV (fig 4a,b) par contre, il est conditionné par le calcium initial $(\mathrm{Ca})$ et par la température finale (Tf); la diminution du Ca est de $13,3 \mathrm{mg}^{\bullet} \mathrm{kg}^{-1}$ par ${ }^{\circ} \mathrm{C}$ (à FCV $=4$ et $\mathrm{Ca}=2,3 \mathrm{~g}^{\bullet} \mathrm{kg}^{-1}$ ), dans tout l'intervalle de variation étudié (fig $4 \mathrm{a}, \mathrm{b}$ ).

Des corrélations entre les minéraux résiduels $(\mathrm{P}$ et $\mathrm{Ca})$ et la $D O$ des lactosérums traités ont été calculées pour l'ensemble des 32 échantillons; elles sont reportées dans le tableau IV. Une corrélation étroite est observée entre $D O$ et $P(r=0,964)$, par contre, il n'y a pas de corrélation entre $D O$ 
et $\mathrm{Ca}$. Le pH final est sous la dépendance du $\mathrm{pH}$ initial (avec une baisse de 0,2 à 0,6 unité selon le $p H i)$, de la température initiale et du calcium initial. $M g$ varie peu, de 100 à $70 \mathrm{mg}^{\circ} \mathrm{kg}^{-1}$ dans tout le domaine des FCV et Ca. K est peu affecté par les traitements.
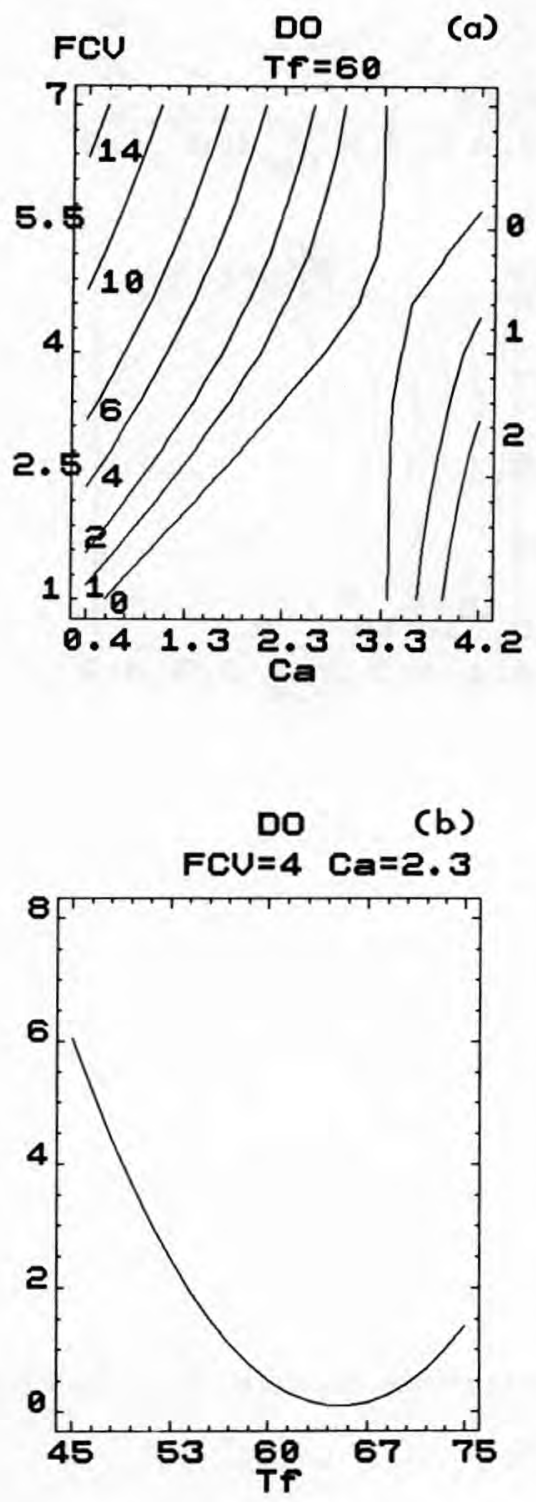

Tableau IV. Coefficients de corrélation. $d P$, $d C a$ : différence entre la teneur du lactosérum initial et du lactosérum après déstabilisation.

Correlation coefficients. $\mathrm{dP}, \mathrm{dCa}$ : difference between initial whey content and whey content after destabilisation.

\begin{tabular}{lrrcrr}
\hline & $D O$ & $C a$ & $P$ & $d P$ & $d C a$ \\
\hline$D O$ & 1,000 & & & & \\
$C a$ & $-0,231$ & 1,000 & & & \\
$P$ & 0,964 & $-0,307$ & 1,000 & & \\
$d P$ & $-0,956$ & 0,361 & $-0,982$ & 1,000 & \\
$d C a$ & $-0,867$ & 0,585 & $-0,905$ & 0,928 & 1,000
\end{tabular}

Une recherche qualitative des phospholipides a révélé la présence de toutes les classes de phospholipides du lactosérum dans les lactosérums clarifiés.

La teneur en MAT du lactosérum est, bien sûr, surtout influencée par le facteur de concentration et assez peu par Tf.

La séparation des constituants protéiques par électrophorèse (fig 5) montre des profils similaires pour les lactosérums non clarifiés $(D O>7,0)$ et complètement clarifiés $(0,1<D O<0,5)$, à l'exclusion de la ré-

Fig 1. Densité optique des lactosérums après traitement (les lignes matérialisent les points ayant la même $D O$ ). a) en fonction du calcium initial $(\mathrm{Ca})$ et du $F C V$, à température finale constante $(T f=60)$; b) en fonction de la température finale $(T f)$, à $F C V=4$ et $C a=2,3 \mathrm{~g}^{\circ} \mathrm{kg}^{-1}$. Sur tous les graphiques, la teneur en calcium initial (Ca) est exprimée en $\mathrm{g} \mathrm{kg}^{-1}$, les teneurs en minéraux du lactosérum clarifié en $\mathrm{mg}^{\circ} \mathrm{kg}^{-1}$, les températures en ${ }^{\circ} \mathrm{C}$ et la MAT en g॰kg ${ }^{-1}$. Optical density of treated wheys: a) versus $\mathrm{Ca}$ and FCV (final temperature $\mathrm{Tf}=60$ ); $\boldsymbol{b}$ ) versus $\mathrm{Tf}$ ( $F C V=4 ; \mathrm{Ca}=2.3 \mathrm{~g}^{\circ} \mathrm{kg}^{-1}$ ). On all the graphs, initial Ca level is in $\mathrm{g}^{\circ} \mathrm{kg}^{-1}$. Ca and other minerals in treated whey in $\mathrm{mg}^{\circ} \mathrm{kg}^{-1}$; temperatures in ${ }^{\circ} \mathrm{C}$. Total nitrogen matter (MAT) in g $\mathrm{kg}^{-1}$. 

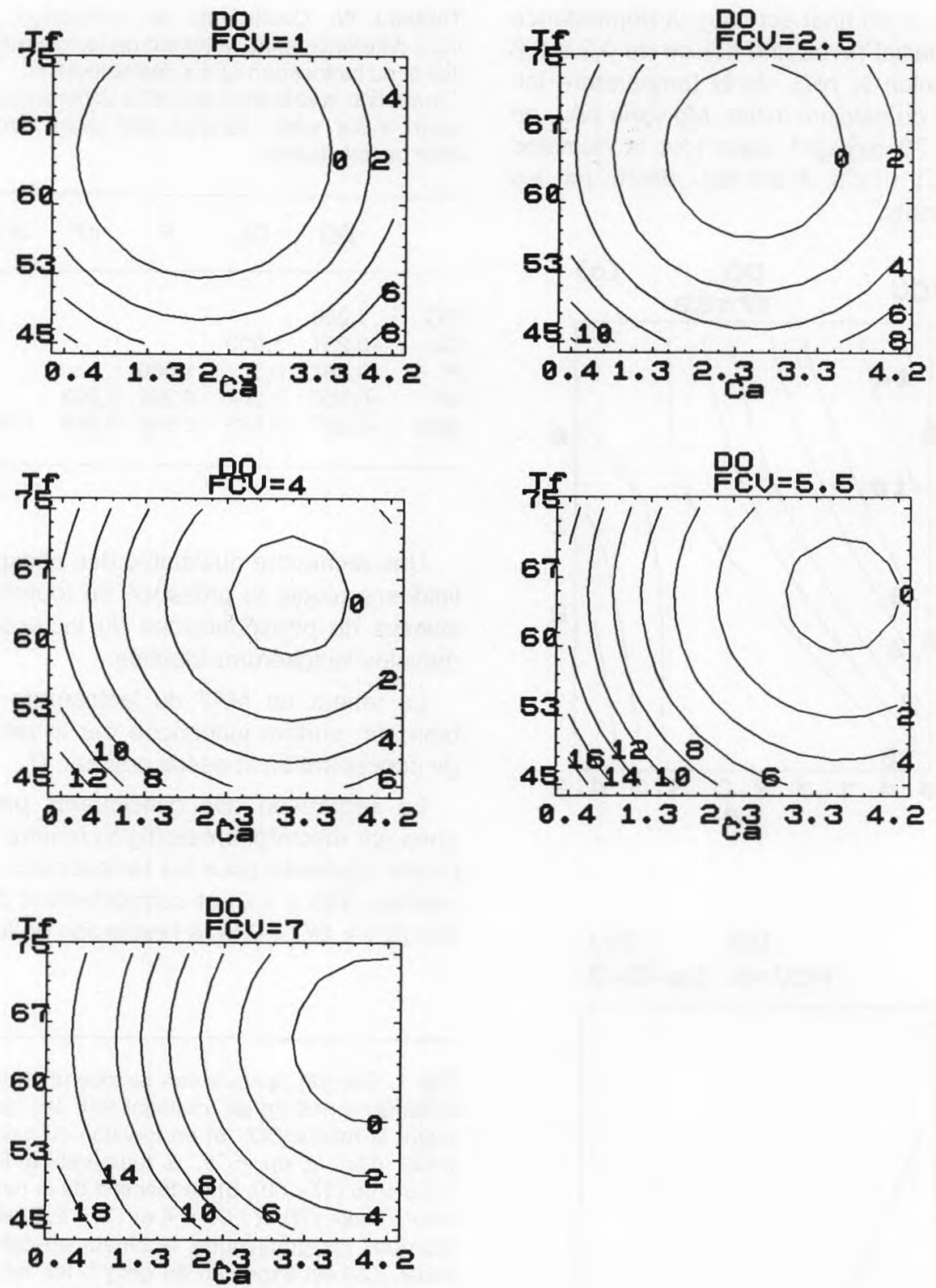

Fig 2. Densité optique des lactosérums après traitement en fonction du Ca et du Tf, une figure pour chaque FCV.

Optical density versus $\mathrm{Ca}$ and $\mathrm{Tf}$ for whey at different $\mathrm{FCV}$. 


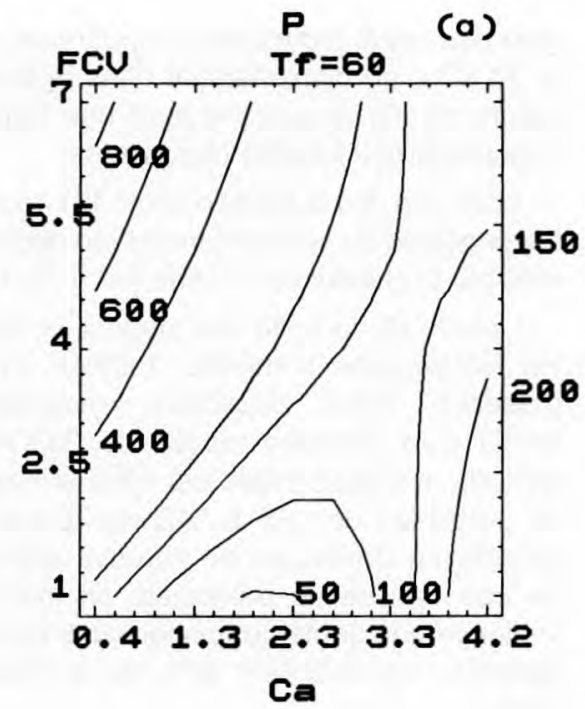

(b)

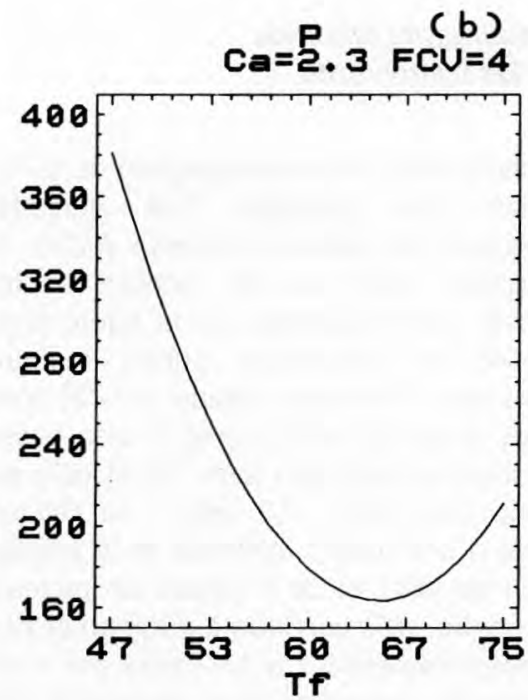

Fig 3. Teneur en phosphore $(P)$ des lactosérum après traitement : a) en fonction du calcium initial $\mathrm{Ca}$ et du $F C V$, à température finale constante $(T f=60)$; b) en fonction de la température

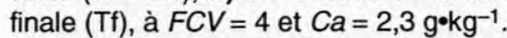

Phosphorus level $(P)$ of treated whey: a) versus initial $\mathrm{Ca}$ level $(\mathrm{Ca})$ and $\mathrm{FCV}, \mathrm{Tf}=60$; b) versus $\operatorname{Tf}(\mathrm{FCV}=4 ; \mathrm{Ca}=2.3)$.
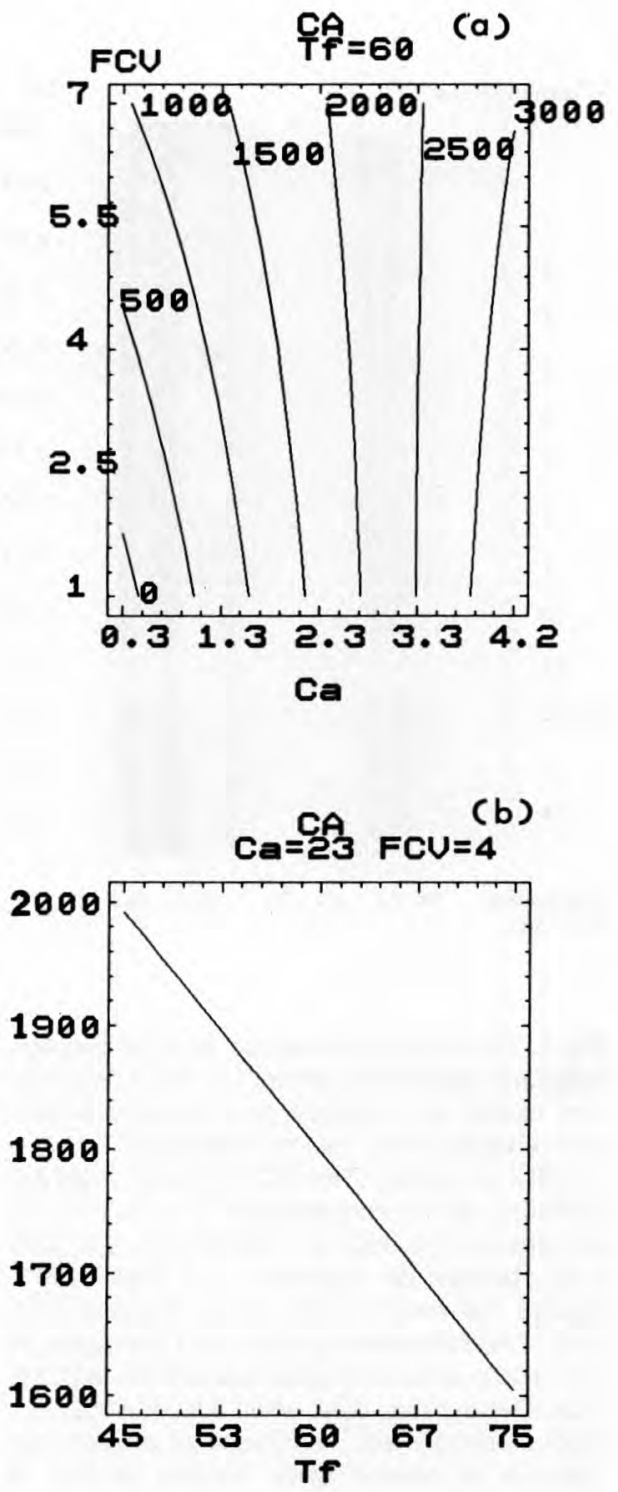

Fig 4. Teneur en calcium ( $\mathrm{Ca}$ ) des lactosérums après traitement : a) en fonction du calcium initial et du $F C V$; b) influence de la température finale sur le calcium final $(\mathrm{Ca})$.

Calcium level (Ca) of treated whey: a) versus initial $\mathrm{Ca}$ level and $\mathrm{FCV}, \mathrm{Tf}=60 ; \boldsymbol{b}$ ) incidence of Tf on final Ca content. 
$n^{\circ}$ échantillons

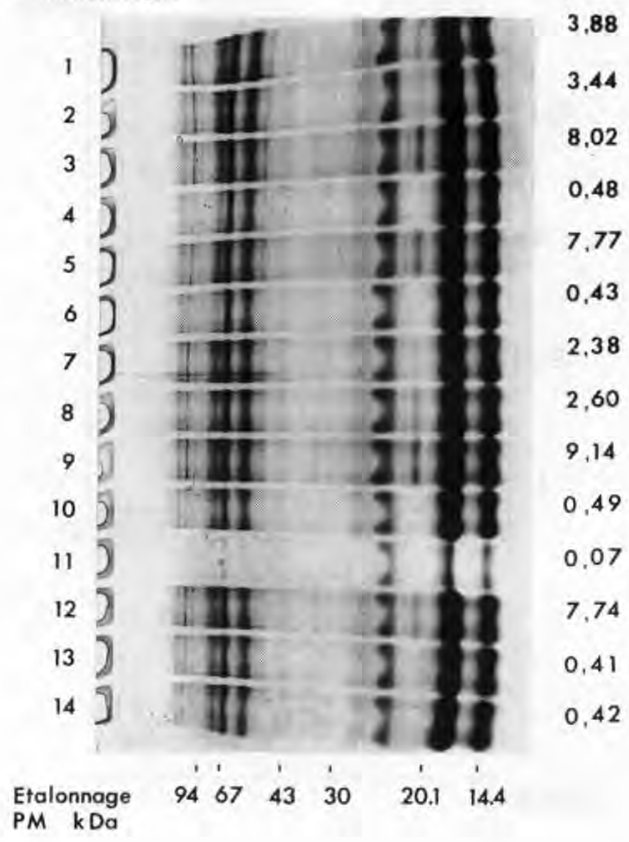

Fig 5. Profil électrophorétique en SDS des lactosérums après clarification. La $D O$ du lactosérum clarifié est reportée pour chaque échantillon analysé. PAE : gel de séparation T $14 \%-$ C 2,8\% en tampon Tris- $\mathrm{HCl} 1,5 \mathrm{~mol}^{\circ} \mathrm{I}^{-1} \mathrm{pH} 8,9$ SDS 0,1 ; gel de concentration T $4,5 \%-\mathrm{C} 1 \%$ en tampon Tris $\mathrm{HCl} 0,5 \mathrm{~mol}^{-1} \mathrm{I}^{-1} \mathrm{pH} \mathrm{6,8} \mathrm{SDS}$ $0,1 \%$; tampon de migration : Tris $0,25 \mathrm{~mol}^{\circ} \mathrm{l}^{-1}$ glycine $1,9 \mathrm{molol}^{-1}$ SDS $0,1 \%$. Fixation TCA $24 \% 1 \mathrm{~h}$ : coloration au bleu de Coomassie R $2500,05 \%$ dans méthanol-eau $(50 / 50)$ fait $12 \%$ TCA. Décoloration dans acide acétique $7 \%$.

SDS electrophoretic separation of proteic constituents of treated whey. Optical density of treated whey is mentioned for each sample analysed. PAE: separation gel T $14 \%-C 2.8 \%$ in 1.5 molor $^{-1}$ Tris- $\mathrm{HCl}$ buffer $\mathrm{pH} 8.9$ SDS $0.1 \%$; stacking gel: T $4.5 \%, C 1 \%$ in 0.5 molor $^{-1}$ Tris$\mathrm{HCl}$ buffer $\mathrm{pH} 6.8 \mathrm{SDS} 0.1 \%$; apparatus buffer: 0.25 molo $^{-1}$ Tris, 1.9 molor $^{-1}$ glycine SDS $0.1 \%$. Fixation $24 \%$ TCA $1 \mathrm{~h}$; staining in $0.05 \%$ Coomassie blue R 250 in 12\% TCA methanol-water (50-50). Destaining in $7 \%$ acetic acid. gion des poids moléculaires apparente, 20 et $30 \mathrm{kDa}$, où disparaissent dans le lactosérum clarifié, respectivement une bande importante et 2 bandes plus ténues.

Dans l'un des échantillons ( $\left.n^{\circ} 11\right)$, toutes les protéines du lactosérum ont été dénaturées par la chaleur car Tf était fixé à $75^{\circ} \mathrm{C}$.

L'étude de la taille des particules dans les lactosérums a montré (tableau V) la présence d'une population majoritaire $(50 \%)$ d'un diamètre moyen de $233 \mathrm{~nm}$; $84 \%$ de la masse totale est sous la forme de particules de 105 à $512 \mathrm{~nm}$. Dans le lactosérum clarifié, on ne retrouve aucune de ces classes de particules; on met en évidence une seule population de $8 \mathrm{~nm}$ de diamètre, représentant $98 \%$ de la masse totale.

\section{Constituants éliminés par les traitements}

La clarification est accompagnée de la formation d'un précipité. Les quantités moyennes de calcium précipité $(\mathrm{dCa})$, de phosphore $(d P)$ et de matière azotée (dMAT) sont reportées sur la figure 6 en fonction de $\mathrm{Ca}$, tous autres facteurs confondus. Pour des teneurs en $\mathrm{Ca}$ comprises entre 0,4 et $2,3 \mathrm{~g}^{\bullet} \mathrm{kg}^{-1}$, une évolution inverse de la DO avec $d P$ et $d C a$ apparaît. Pour $\mathrm{Ca}>2,3 \mathrm{~g}^{\circ} \mathrm{kg}^{-1}$, la $D O$ est stable à une valeur minimale et la précipitation de MAT et de $P$ atteint un plateau. Par contre, $d C a$ continue à augmenter proportionnellement à $\mathrm{Ca}$. Le calcul des coefficients de corrélation pour l'ensemble des résultats montre la liaison de $D O$ avec $d P$ $(r=-0,956)$ et avec $d C a(r=-0,867)$, et en conséquence de $d P$ et $d C a$ (tableau IV).

La précipitation du $P(d P)$ est également sous la dépendance de la concentration du lactosérum (fig 7a). Le Ca précipite d'autant moins de $\mathrm{P}$ que le FCV est élevé. Cor- 
Tableau V. Taille des particules dans le lactosérum.

Size of particulate material in whey.

\begin{tabular}{lrrrr}
\hline Lactosérum brut $(\mathrm{n}=6)$ & & & & \\
Diamètre moyen $(\mathrm{nm})$ & 105 & 233 & 512 & 1950 \\
$\mathrm{~s}$ & 28 & 55 & 150 & 400 \\
\% de la masse totale & 19 & 50 & 15 & 3 \\
& & & & \\
Lactosérum clarifié $(\mathrm{n}=5)$ & & & & 1450 \\
Diamètre moyen $(\mathrm{nm})$ & 8 & 100 & & 370 \\
$\mathrm{~s}$ & 4 & 45 & & 1 \\
$\%$ de la masse totale & 98 & 1 & & \\
\hline
\end{tabular}

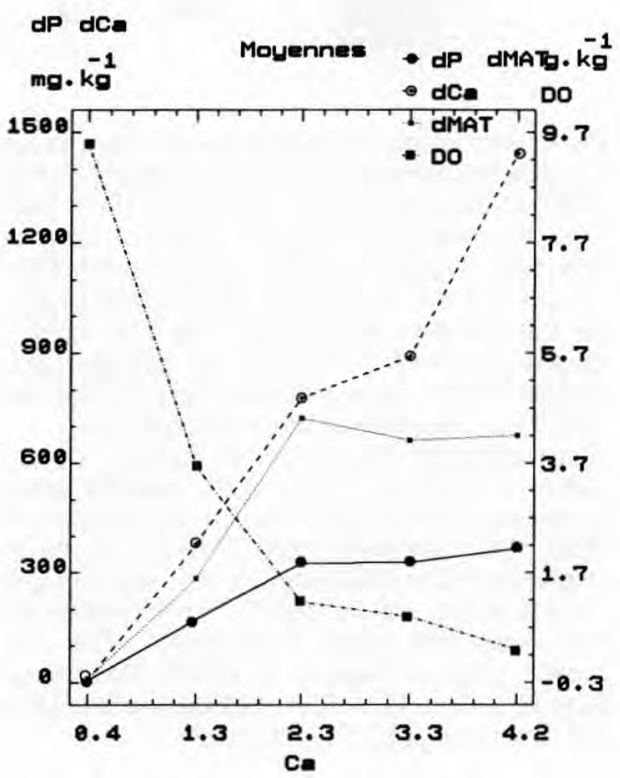

Fig 6. Valeurs moyennes pour l'ensemble des essais, de $d P, d C a, d M A T$ et $D O$, en fonction du calcium total $(\mathrm{Ca})$.

Mean values of precipitated fractions, $\mathrm{dP}, \mathrm{dCa}$, dMAT, and optical density versus initial $\mathrm{Ca}$.

rolairement, une teneur plus élevée en $\mathrm{Ca}$ est nécessaire pour précipiter la quantité maximale de $P: 1,3 \mathrm{~g}^{\circ} \mathrm{kg}^{-1}$ de Ca à $F C V 1$; $4,2 \mathrm{~g}^{\circ} \mathrm{kg}^{-1}$ de $C a$ à $F C V 7$. En revanche, la quantité maximale de $\mathrm{P}$ précipitable ne dé- pend pas du FCV : elle est de l'ordre de 350 à $400 \mathrm{mg}^{\circ \mathrm{kg}^{-1}}$ quel que soit le $F C V$.

La quantité de calcium précipité $(\mathrm{dCa})$ dépend peu du FCV (fig $7 \mathrm{~b}$ ), surtout lorsque la teneur en Ca est élevée, par contre, $d C a$ augmente proportionnellement à $\mathrm{Ca}$.

La précipitation de matière azotée est proportionnelle au FCV. Elle est de l'ordre de $0,9 \mathrm{~g} \mathrm{~kg}^{-1}$ pour le lactosérum. Une électrophorèse par charge du précipité de clarification (fig 8) met en évidence de nombreux constituants protéiques. II a été possible de les identifier, grâce à leur référence front, aux constituants des protéoses peptones décrits par Andrews et Alichanidis (1983) dans le même système électrophorétique de séparation. Le constituant $n^{\circ} 14$ semble le plus abondant; il correspond à la fraction "PP5" ou BCN5P, le $n^{\circ} 19$ au BCN1P, le $n^{\circ} 38$ au BCN4P, les autres constituants du précipité correspondent aux constituants mineurs des protéoses peptones du lait.

\section{DISCUSSION}

Au cours de la clarification, l'évolution de la $D O$ du lactosérum est associée à la précipitation du P (fig 1a, 3a) et du Ca avec des coefficients de corrélation respectifs de $-0,956$ et $-0,867 ; 80 \%$ du phosphore total 

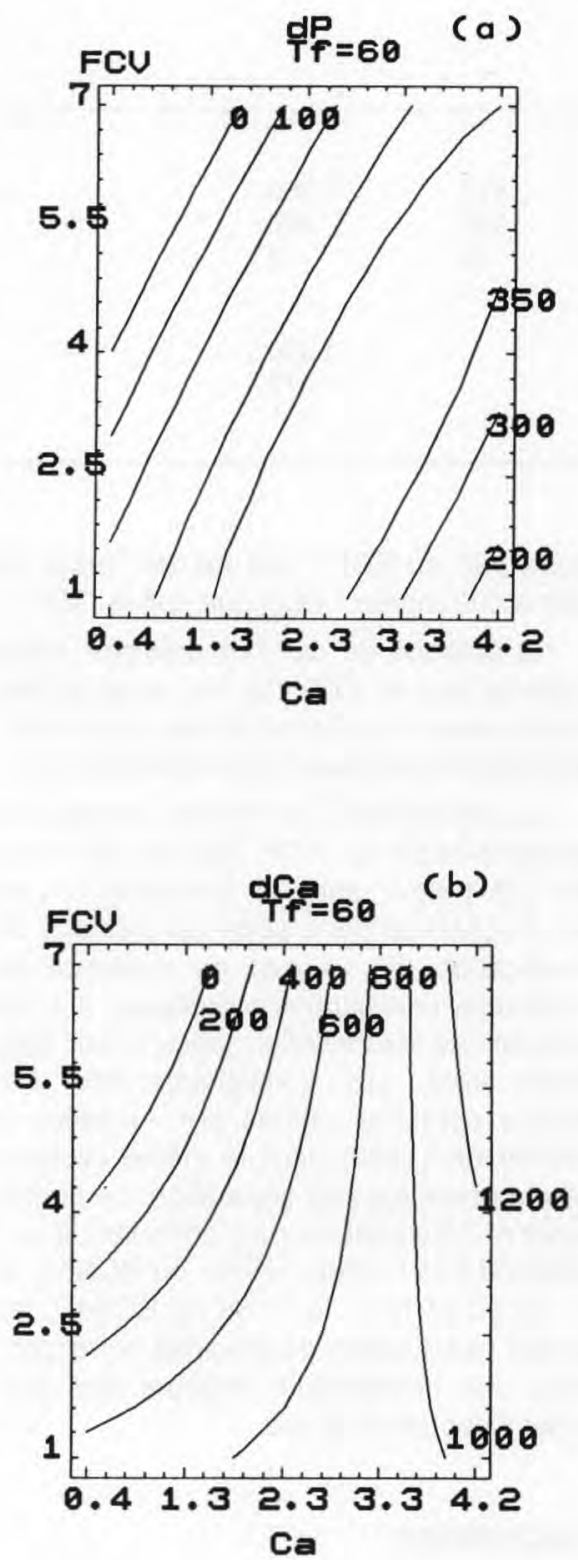

Fig 7. Phosphore et calcium précipités (dP et $\mathrm{dCa}$ ) en fonction du calcium initial (Ca) et du FCV à $T f=60$.

Precipitated calcium (dCa) and phosphorus (dP) versus initial calcium (Ca) and $F C V$. Tf $=60$.

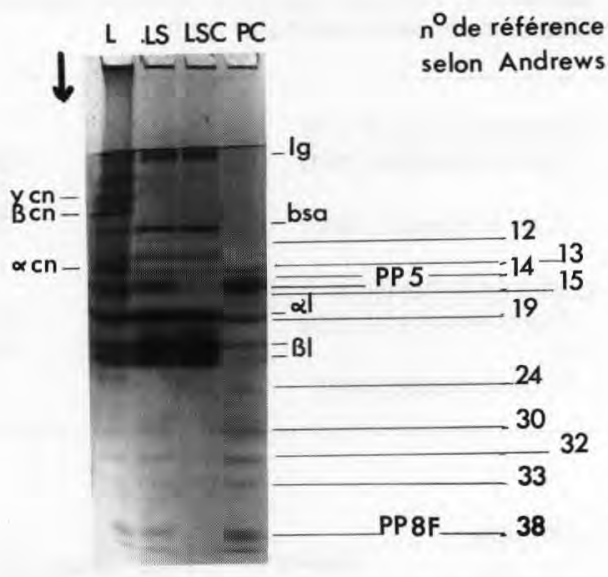

Fig 8. Profil électrophorétique par charge d'un lait (L), d'un lactosérum (LS), d'un lactosérum clarifié (LSC) et d'un précipité de clarification (PC). PAE : gel de préparation T $12,5 \%$ - C $4 \%$ en tampon Tris $\mathrm{HCl} 0,38$ molol-1 $^{-1} \mathrm{pH} 8,9$; gel de concentration T 4,2\% - C $5 \%$ en tampon Tris $\mathrm{HCl} 0,06$ molol $^{-1}$ $\mathrm{pH} 6,8$. Tampon de migration Tris $0,25 \mathrm{molol}^{-1}$, glycine $1,9 \mathrm{~mol}^{-1}$. Coloration au bleu de Coomassie $0,05 \%$ dans méthanol-eau $(50 / 50)$ fait $12 \%$ TCA. Décoloration acide acétique $7 \%$.

Electrophoretic separation of proteic constituents of milk (L), whey (LS), clarified whey (LSC) and precipitated fraction on clarification (PC). PAE: Separation gel: $T 12.5 \%, C 4 \%$ in 0.38 molor $^{-1}$ Tris HCl buffer pH 8.9; stacking gel: $\mathrm{T} 4.2 \%, \mathrm{C} 5 \%$ in 0.06 molo $^{-1}$ Tris $\mathrm{HCl}$ buffer $\mathrm{pH}$ 6.8. Apparatus buffer $0.25 \mathrm{molot}^{-1}$ Tris, 1.9 molot ${ }^{-1}$ glycine. Staining in $0.05 \%$ Coomassie blue $R 250$ in $12 \%$ TCA methanol-water (5050). Destaining in $7 \%$ acetic acid.

du lactosérum sont précipités lors de ce traitement.

Le $\mathrm{P}$ total du lactosérum $(P)$ est composé de phosphore minéral $\left(P_{m}\right)$ et de phosphore organique $\left(P_{o}\right)$, ce dernier étant luimême constitué par des esters phosphate $\left(P_{e}\right)$ et par du phosphore protéique $\left(P_{p}\right)$ :

$$
P=P_{\mathrm{m}}+P_{\mathrm{o}}-\text {, où } P_{\mathrm{o}}=P_{\mathrm{e}}+P_{\mathrm{p}}
$$


Le phosphore total $(P)$ du lactosérum était égal à $440 \mathrm{mg}^{\circ} \mathrm{kg}^{-1}$; le $P_{e}$ peut être estimé à $90 \mathrm{mg}^{\bullet \mathrm{kg}^{-1}}$ (Walstra et Jenness, 1984). Le $P_{p}$ fixé sur des protéines ou des complexes macromoléculaires retenus par ultrafiltration a été évalué, d'après nos résultats, à $20 \mathrm{mg}^{\circ} \mathrm{kg}^{-1}$. En conséquence, $\mathrm{P}_{\mathrm{o}}=90+20=110 \mathrm{mg}^{\circ} \mathrm{kg}^{-1}$ et donc $P_{m}=$ $330 \mathrm{mg} \cdot \mathrm{kg}^{-1} . P_{\rho}$ comprend plusieurs fractions d'origine différente : 1) les peptides phosphorylés issus de l'hydrolyse des caséines, fractions de la caséine $\beta$ libérées par la plasmine (BCN5P, BCN1P, $\mathrm{BCN} 4 \mathrm{P})$, caséinomacropeptide (CMP) de la caséine $\kappa(1 \mathrm{P})$ libéré par la présure, fractions peptidiques diverses libérées par les microrganismes, 2) la fraction membranaire des globules gras, contenant le $\mathrm{P}$ constitutif des phospholipides.

La clarification entraîne la précipitation des peptides phosphorylés de la caséine $\beta$ que nous avons mis en évidence lors de l'électrophorèse en SDS sous forme d'un constituant de poids moléculaire apparent de $20 \mathrm{kDa}$ (Mati et al, 1991). Par contre, nous ne connaissons pas le devenir du CMP. Les membranes de globules gras semblent également être précipitées. En effet, on sait qu'elles sont dans le lactosérum sous forme de particules de 30-200 $\mathrm{nm}$ de diamètre (Webb et al, 1978) et nous avons montré que la clarification élimine toutes les particules du lactosérum ayant un diamètre $>100 \mathrm{~nm}$. Nos résultats n'en apportent pas la preuve sur le plan chimique. En effet, les électrophorèses n'ont pas été réalisées dans des conditions permettant la mise en évidence de leurs constituants protéiques spécifiques.

Par ailleurs, les phospholipides, fractions caractéristiques de ces structures, ont bien été mis en évidence dans les lactosérums bruts, mais également dans les lactosérums clarifiés. Baumy et al (1990) avaient déjà montré que l'on retrouve une partie de phospholipides dans le lactosérum clarifié.
II est possible qu'au cours des traitements appliqués, une partie des membranes de globules gras ait été fractionnée pour donner des vésicules de 10 à $50 \mathrm{~nm}$ de diamètre, telles que les décrivent Walstra et Jenness (1984). Elles constitueraient la population que nous avons mise en évidence dans le lactosérum clarifié et y expliqueraient la présence de phospholipides.

Ainsi, la plupart des fractions du $P_{p}$ sont précipitées lors de la clarification.

Hayes et al (1974) ont montré que le $P_{m}$ de la phase soluble (saturée à $37^{\circ} \mathrm{C}$ ) précipitait lors du traitement thermique de clarification du lactosérum.

Nous n'avons pas dosé les esters du phosphate et nous ne savons pas quelle est leur partition au cours de la clarification, cependant, étant donné leur grande solubilité, il est probable qu'ils restent en solution dans le lactosérum clarifié.

Le phosphore précipité par clarification comprend donc la quasi-totalité du $P_{m}$ et du $P_{p}$, soit $350 \mathrm{mg}^{\circ \mathrm{kg}^{-1}}$ au maximum. Le phosphore résiduel du lactosérum clarifié contient le $P_{e}$ et une faible proportion du $P_{m}$, pour satisfaire au produit de solubilité, soit au total 90-150 $\mathrm{mg}^{\bullet \mathrm{kg}^{-1}}$ d'après nos résultats, quel que soit le FCV du lactosérum initial.

La teneur en $P$ précipitable serait donc le facteur limitant la précipitation du phosphate de calcium.

Les courbes de la figure 6 montrent l'évolution de $d P, d M A T$ ainsi que celle de la $D O$. On remarque qu'à tous les stades de la précipitation, l'évolution de $d P$ et dMAT est liée; cela semble indiquer une coprécipitation de $P_{m}$, de $P_{p}$ et de la MAT associée. Si un processus en 2 temps se déroulait, par exemple une précipitation du $P_{m}$ suivie de celle de $P_{p}$, un tel mode de représentation le ferait apparaitre.

Nos résultats permettent de conclure que le traitement appliqué entraîne la for- 
mation d'un phosphate de calcium mixte insoluble constitué de phosphate minéral et de phosphate organique entraînant les fractions protéiques et lipidiques. Ce sel, formé dans des conditions de précipitation rapide serait de type colloïdal (Hayes et al, 1974). La quantité de MAT associée est de $0,9 \mathrm{~g} \mathrm{~kg}^{-1}$ pour le lactosérum et augmente proportionnellement au FCV, ce qui représente $10 \%$ de la MAT mise en œuvre. L'agrégation des constituants est progressive en fonction de la teneur en Ca.

L'induction de la précipitation nécessite une teneur en calcium élevée. La quantité utile varie linéairement avec la concentration en protéines du lactosérum, de 0,7 à 2,9 $\mathrm{g}^{\circ} \mathrm{kg}^{-1}$ de Ca pour des lactosérum de FCV 1 à 5,5 (MAT 8,7-37,7 $\left.\mathrm{g}^{\circ} \mathrm{kg}^{-1}\right)$; puis elle se stabilise à $3,3 \mathrm{~g} \bullet \mathrm{kg}^{-1}$ de Ca pour les lactosérum de FCV 5,5 à 7 (MAT 37,7 à $\left.50,8 \mathrm{~g}^{\circ} \mathrm{kg}^{-1}\right)$. Pourquoi une teneur en calcium aussi élevée est-elle nécessaire, alors que la quantité précipitée est de 500-1000 $\mathrm{mg}^{\circ} \mathrm{kg}^{-1}$, pour une clarification complète? II semble probable que la présence des peptides phosphorylés augmente le produit de solubilité du sel mixte et augmente aussi la stabilité du phosphate de calcium minéral, comme l'ont montré Schmidt et al (1987).

Les traitements appliqués lors de nos expérimentations ont pu aboutir à la clarification de tous les lactosérums étudiés, de FCV1 à FCV7 (MAT 8,7-50,8 g*kg ${ }^{-1}$ ). La figure 2 montre les plages où les facteurs sont optimaux. Les 5 facteurs n'ont pas tous la même importance pour la réalisation de la clarification. Les facteurs $T i$ et $p H i$ ont été trouvés de très peu de poids dans l'intervalle de variation des facteurs étudié. La teneur en calcium a une influence prépondérante. Une augmentation de la température finale entre 45 et $60^{\circ} \mathrm{C}$ favorise la clarification et la précipitation du Ca et du P.
La clarification apparaît comme un phénomène progressif d'agrégation des constituants phosphorés du lactosérum en association avec du calcium. Pour certains niveaux des facteurs, nous avons obtenu des clarifications partielles, avec des valeurs de $D O$ intermédiaires et une précipitation partielle des minéraux, par exemple avec des teneurs en calcium sousoptimales.

Les graphiques des figures 2 et 3 représentent un tracé pour la valeur $D O=0$, correspondant à la clarification totale prévue par le calcul à partir de nos résultats expérimentaux. Or, nos résultats ont montré que la valeur minimale de la $D O$ après clarification est de 0,07 pour le lactosérum et de 0,5 au $F C V 7$, soit une valeur de 0,07 pour une unité $F C V$. Pratiquement, la clarification des lactosérums peut donc être considérée comme totale après des conditions de traitement où ces valeurs sont atteintes.

II est probable que pour des niveaux intermédiaires des facteurs, conduisant à des clarifications partielles, les conditions de centrifugation choisies pour l'élimination du précipite aient une influence sur la turbidité résiduelle du lactosérum clarifié. Dans notre travail, une centrifugation à basse vitesse a été utilisée, mais il est possible qu'avec des forces centrifuges plus élevées, une élimination plus complète des particules de faible taille aurait été obtenue et les zones de clarification auraient eu des contours plus larges sur les représentations de la figure 2 . De même, si on envisage la séparation du précipité à l'aide d'un autre procédé, la microfiltration sur membrane, par exemple, les contours des conditions optimales seront à redéfinir et, de plus, il faudra tenir compte des performances hydrodynamiques. C'est ce que nous nous proposons d'étudier dans un prochain travail. 


\section{REMERCIEMENTS}

Nous remercions $R$ Pannetier pour les analyses de MAT, JL Maubois et G Daufin pour leur lecture critique du manuscrit.

\section{REFERENCES}

Andrews AT (1983) Proteinases in normal bovine milk and their action on caseins. J Dairy Res 50, 45-55

Andrews AT, Alichanidis E (1983) Proteolysis of caseins and the proteose peptone fraction of bovine milk. J Dairy Res 50, 275-290

Baumy JJ, Gestin L, Fauquant J, Boyaval E, Maubois JL (1990) Technologie de purification des phospholipides du lactosérum. Process 1047, 29-33

Bligh EG, Dyer WJ (1959) A rapid method of total lipids extraction and purification. Can $J$ Biochem Physiol 37, 911-917

Box GEP, Wilson KB (1951) On the experimental attainment of optimum conditions. J R Stat $\operatorname{Soc} B 13,1-45$

Box GEP, Hunter JS (1957) Multifactor experimental designs for exploring response surfaces. Ann Math Stat 28, 195-241

Daufin G, Labbé JP, Quémerais A, Michel F (1991) Fouling of an inorganic membrane during ultrafiltration of defatted whey protein concentrates. Neth Milk Dairy J 45, 259-272

Daufin G, Michel F, Merin U (1992a) Ultrafiltration of defatted whey: influence of some physicochemical characteristics. Aust $J$ Dairy Technol (sous presse)

Daufin G, Michel F, Merin U (1992b) Study of ultrafiltration of defatted whey protein concentrates (WPC) withdrawn from an industrial plant. Lait 72, 185-199

de Wit JN, Klarenbeek G, de Boer R (1978) Une méthode simple pour la clarification du lactosérum. XX $X^{\ominus}$ Congrès Int Brèves Commun F 935

Ducruet V, Degas C, Lamberet G (1990) Composition lipidique d'un sous produit de lactosérum de caséinerie. Lait 70, 117-126

Durier C, Kobilinski A (1989) APOLOR : une nouvelle vision de la régression polynominale. XX|e Journées de Statistiques, Rennes
Fauquant J, Vieco E, Brulé G, Maubois JL (1985a) Clarification des lactosérum doux par agrégation thermocalcique de la matière grasse résiduelle. Lait $65,1-20$

Fauquant J, Pierre A, Brulé G (1985b) Clarification du lactosérum acide de caséinerie. Tech Lait 1003, 37-41

FIL-IDF (1982) Determination de la teneur en phosphore total dans le fromage. Norme $\mathrm{n}^{\circ} 33 \mathrm{~B}$

Folch J, Lees MB, Sloane-Stanley GH (1957) A simple method for the isolation and purification of the total lipids from animal tissues. J Biol Chem 226, 497-509

Hayes JF, Dunkerley JA, Muller LL, Griffin AT (1974) Studies on whey processing by ultrafiltration. II: Improving permeation rates by preventing fouling. Aust J Dairy Technol 29, 132-140

Jenness R, Koops J (1962) Preparation and properties of a salt solution which simulates milk ultrafiltrate. Neth Milk Dairy $J$ 16, 153164

Laemmli UK (1970) Cleavage of structural properties during the assembly of the head of bacteriophage T4. Nature (Lond) 227, 680685

Lee DN, Merson RL (1976) Prefiltration of cottage cheese whey to reduce fouling of ultrafiltration membranes. J Food Sci 41, 403410

Mangold HK, Kammereck R (1962) New methods of analysing industrial aliphatic lipids. J Am Oil Chem Soc 39, 201-206

Marshall KR, Kavanagh JA, Parkin MF (1974) Ultrafiltration de lactosérum de caséine acide. XIXe Int Dairy Congr Brèves Commun $1 \mathrm{~F}, 849$

Mati A, Girardet JM, Xenakis D, Linden G (1991) Isolement et caractérisation de la fraction hydrophobe des protéoses peptones des laits bovins, ovins et caprins. Lait 71, 259-273

Maubois JL, Pierre A, Fauquant J, Piot M (1987) Industrial fractionation of main whey proteins. Bull Fed Int Lait 212, 154-159

Noël Y, Durier C, Lehembre N, Kobilinsky A (1991) Étude multi-factorielle de la coagulation mixte du lait analysée par viscoélasticimétrie. Lait 71, 15-39

Schmidt DG, Both $P$, Visser S, Slangen KJ, van Rooijen PJ (1987) Studies on the precipita- 
tion of calcium phosphate. II. Experiments in the $\mathrm{pH}$ range 7.3 to 5.8 at 25 and $50^{\circ} \mathrm{C}$ in the presence of additives. Neth Milk Dairy $J$ $41,121-136$

Walstra P, Jenness R (1984) Dairy chemistry and physics. John Wiley and Sons, New York
Wasen I, Lehmann H, Westphalia Separator (1989) Procédé de déphospholipidation du petit lait. Brevet Fr 2625649 A1

Webb BH, Johnson AH, Alford JA (1978) Fundamentals of dairy chemistry. The Avi Publishing Company, Inc Westport, Connecticut 